\title{
On the burning and microexplosion of collision-generated two-component droplets: miscible fuels
}

\author{
C.H. Wang ${ }^{\mathrm{a}, *}$, W.G. Hung ${ }^{\mathrm{a}}$, S.Y. Fu ${ }^{\mathrm{a}}$, W.C. Huang ${ }^{\mathrm{a}}$, C.K. Law ${ }^{\mathrm{b}}$ \\ ${ }^{a}$ Department of Mechanical Engineering, National Taiwan University, Taipei, Taiwan \\ ${ }^{\mathrm{b}}$ Department of Mechanical and Aerospace Engineering, Princeton University, Princeton, NJ 08544, USA
}

Received 26 July 2002; received in revised form 18 February 2003; accepted 25 March 2003

\begin{abstract}
The combustion and microexplosion of freely falling two-component droplets, generated either independently or through the collision or merging of two droplets of the two fuels have been studied experimentally. The results show that the non-disruptive combustion characteristics, including the ignition delay, the flame shrinkage and the burning rates are largely similar for droplets generated with these two different modes, hence indicating the efficiency of mixing through the internal motion produced when droplets coalesce. Microexplosion induced by internal superheating and hence nucleation, however, was only observed for the collision-generated droplets, and is believed to be initiated by the air bubbles entrained during a collision. The potential importance of bubble entrainment during droplet and spray generation on spray atomization and burnout is emphasized. () 2003 The Combustion Institute. All rights reserved.
\end{abstract}

Keywords: Two-component droplets; Droplet collision; Microexplosion

\section{Introduction}

Motivation for the present investigation came from the confluence of two phenomena relevant to droplet and spray combustion, namely the combustion and microexplosion of single droplets of multicomponent fuels, and the coalescence and bouncing of two colliding droplets. Regarding the background for multicomponent droplet combustion, we note that $[1,2]$ the study is of significant technical interest because most practical liquid fuels are blends of many chemicals each characterized by its own physical-chemical properties, such as volatility, diffusivity, and reactivity. In particular, it is well established that the dominant factor governing the gasification of

\footnotetext{
* Corresponding author. Tel.: +02-23634254; fax: $+02-23631755$.

E-mail address: chinghua@ccms.ntu.edu.tw (C.H. Wang)
}

a multicomponent droplet is the exceedingly slow rate of liquid-phase mass diffusion relative to those of the droplet surface regression and liquid-phase thermal diffusion [3]. Consequently, as a droplet gasifies, the volatile components are initially preferentially gasified from the droplet surface, while the composition of the droplet's interior remains practically unchanged. A concentration gradient is then set up within the droplet in such a manner that its composition changes from that of the interior, and hence initial, state to that of the surface which is more concentrated with the less volatile components. Because the less volatile components have higher boiling points, the droplet therefore sustains an equilibrium vaporization temperature higher than that based on the initial composition.

This diffusion-limited combustion mechanism has several implications. First, because gasification is initially dominated by the more volatile components, ignition of the droplet should also be affected more 


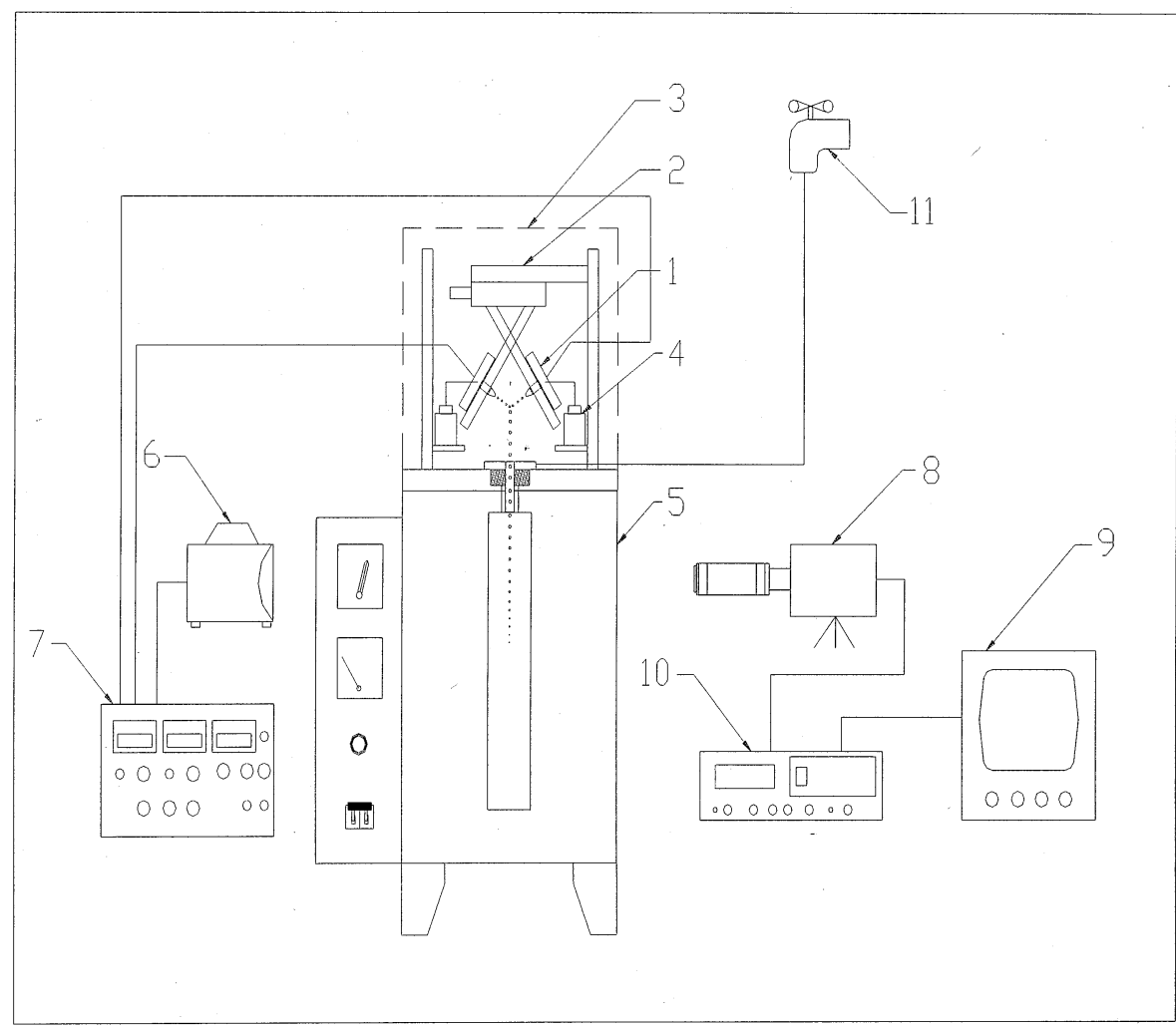

\section{1.droplet generators 2.collision device 3.air tight chamber 5.combustion chamber 6.stroboscope 7.electronic controller 4.fuel reservoirs 9.monitor 10.recorder 11.circulating cooling water}

Fig. 1. Schematic of the experimental setup.

by the reactivity of these components. Second, since the droplet undergoes substantial heating as the less volatile components start to leave the droplet, the gasification rate of the droplet and hence the flame size should momentarily decrease during this transitional heating period. Third, after establishment of the concentration boundary layer, the droplet gasification rate should remain fairly steady. Fourth, the subsequent high surface and hence droplet temperature relative to the value for gasification of the interior implies that the droplet interior can potentially be heated to the limit of superheat. At this state, instantaneous internal gasification would take place, leading to violent rupturing of the droplet-a phenomenon termed microexplosion. These unique characteristics of the combustion of a multicomponent droplet have been largely verified experimentally [4-8], although it has been noted [6] that the occurrence of microexplosion seems to depend on how the droplet was generated. We shall present additional experimental evidence suggesting the possible cause for such dependence, which is of fundamental interest and is also practically important.

Regarding the collision of droplets, we note that since the droplet concentration is very large immediately downstream of a spray injector, it has been suggested that droplets frequently collide [9]. Further studies [10-12] have shown that when two droplets impinge on each other, the pressure buildup in the inter-droplet space causes the otherwise convex droplet surfaces to become concave. Consequently the locations of closest approach for the droplets are the rims forming the edges of these indentations. Thus, a gas bubble would be entrained in the merged mass in the event that the droplets do merge upon contact (at the rims). It has also been shown computationally [12] that intense motion is generated within the interior of the merged mass upon coalescence, leading to 


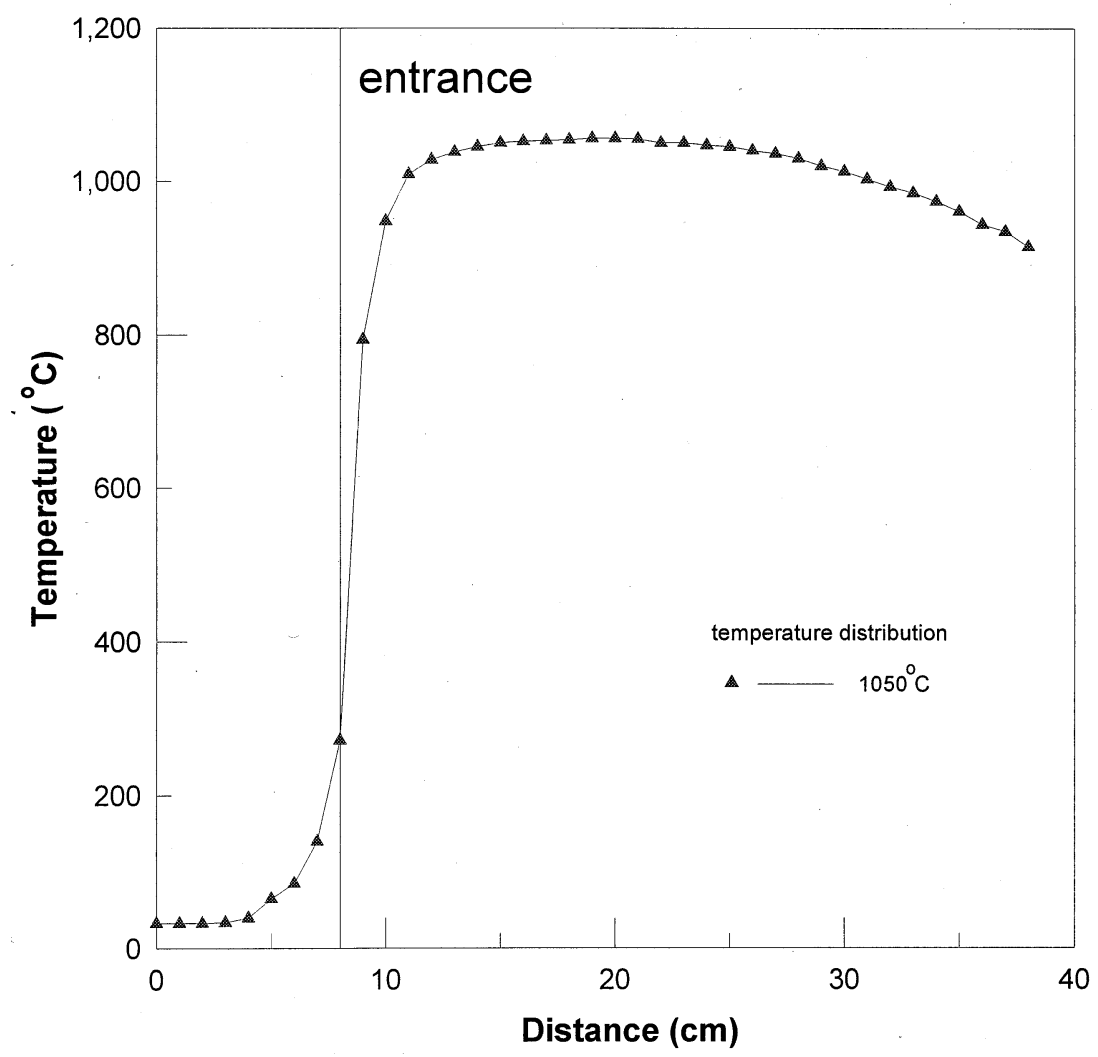

Fig. 2. Centerline temperature profile of the connection channel and furnace.

dissipation of the excess surface energy and homogenization of the liquid's interior. Such motion could also disrupt the initially entrained gas bubble into smaller pieces.

Thus, it is important to investigate the combustion characteristics of multicomponent droplets formed through droplets colliding. Because of the different vaporization histories experienced by different droplets in practical situations, it is reasonable to expect that two colliding droplets with the same initial composition might well have different compositions when they collide and merge. It is also of relevance to the impinging type of atomization involving two reactants. Thus, it is of interest to explore the potential differences in the ignition and burning characteristics between, say, a two-component droplet formed through the merging of two single-component droplets of different fuels from one that is originally prepared with such a composition. The controlling factor here is the rate of mixing through the internal flow generated upon merging. Clearly for a sufficiently rapid mixing rate the gasification of the merged droplet should be similar to that of the original single droplet.

We further note that, regardless of the rate of mixing, there is a major difference between droplets generated through the two different mechanisms described above in that the merged droplet would have one or more gas bubbles entrained in its interior. While the presence of the bubbles is not expected to affect a droplet's gasification and burning characteristics, they could initiate nucleation of the superheated liquid elements in the interior of the droplet. As such, it is possible that the merged droplet could micro-explode easier. The practical implication of such a possibility in enhanced atomization is quite apparent.

\section{Experimental apparatus and test procedure}

Figure 1 shows the experimental apparatus. Except for the collision device, the rest of the setup is similar to that used previously $[7,8]$. Specifically, an ink-jet printing injector with a circular piezoelectric crystal plate was used to generate freely falling droplets that are spatially and temporally stable over extended periods of time. To study the collision of the droplets, two such injectors with the same pulse rate, but different pulse widths and amplitudes, were used. 


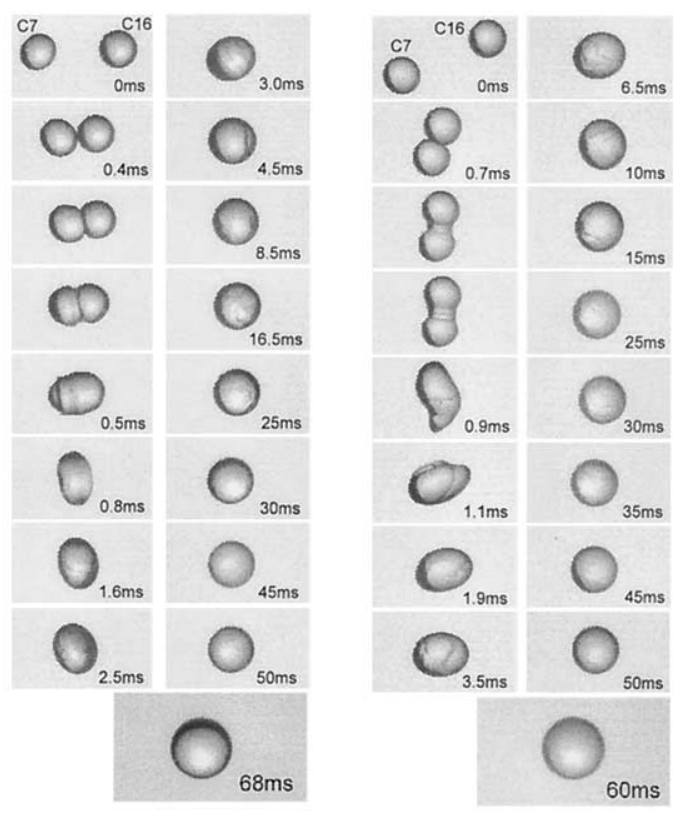

$\mathrm{C}_{7} \mathrm{H}_{16} \quad 0.263 \mathrm{~mm}$ $\mathrm{C}_{16} \mathrm{H}_{34} 0.267 \mathrm{~mm}$
0.263

0.267

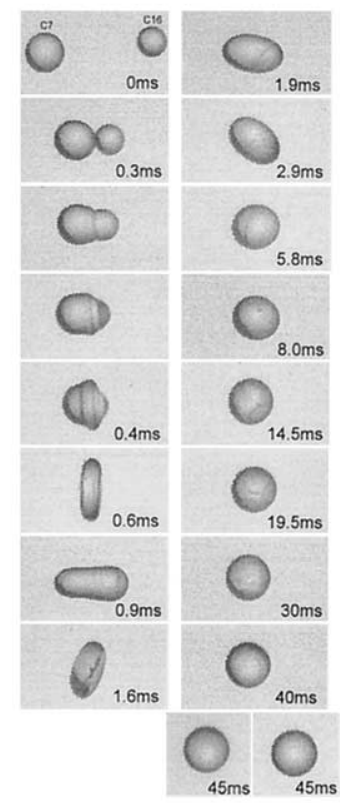

0.280

0.206

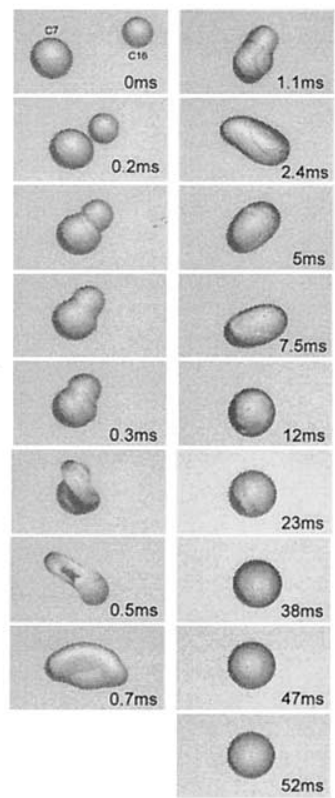

0.280

0.206

\section{Head-on-collision Graze-collision $\mathrm{Vr} \sim 0.65 \mathrm{~m} / \mathrm{s}$}

\section{Head-on-collision Graze-collision $\sim 1.5 \mathrm{~m} / \mathrm{s}$}

Fig. 3. Photographic images showing various collision sequences resulting in droplet coalescence.

The two droplet streams were manipulated to collide in the desired manner by adjusting their trajectories on the same plane, varying the phase difference and/or the relative positions of the two generators. By seating the generation assembly on a 2-axes translator, the merged droplets can be adjusted to fall through a small vertical channel connected to a high temperature, oxidizing combustion chamber. The high-temperature furnace used nickel-chromium wire for heating, fire brick for wall insulation, and had a temperature range from room temperature to $\sim 1050^{\circ} \mathrm{C}$ controlled by a thermostat and monitored by a thermocouple. Circulating water was used to separate the droplet generation device from the high temperature environment.

The droplet size was varied from $100-400 \mu \mathrm{m}$, mainly by changing the size of the glass nozzle of the generator. The composition of the merged droplets was determined by the volume ratio of the two colliding droplets. A strobe light synchronized with the droplet generators, with variable phase lags, was used to freeze the moving droplets for observation and photography at various stages of the droplet lifetime. Enlarged droplet images were recorded on a CCD camera with a Bausch \& Lomb Mono-zoom-7 long focus microscopic lens. The premixed and collisiongenerated droplets became basically spherical after some relaxation time to damp out the oscillation of the merged mass upon coalescence. The uncertainty in the determination of the droplet size was $\sim 2 \%$, mainly from reading the boundary of the droplet image.

Using this apparatus, the droplet ignition delay, burning time and rate, and also the state of microexplosion were investigated. Ignition delay was defined as the time interval from the instant at which the droplet was exposed to the high-temperature environment to the instant when a visible flame was observed. Experimental resolution of the time scale of the electronic device was $\sim 0.04 \mathrm{~ms}$, while the uncertainty of defining the ignition state was estimated to be less than $1 \mathrm{~ms}$. The droplets were generated at $\sim 30 \mathrm{~Hz}$, and were separated in excess of 100 diameters such that essentially they did not interact, as noted previously [13]. Furthermore [13], while the 
ignition delay decreased with increasing relative velocity between the droplet and the environment, the effect was minor for the experimental conditions of the present investigation.

Figure 2 shows the temperature profile along the center of the connection channel and the furnace. It is seen that there exists a certain distance over which the center temperature increases and eventually reaches the final temperature. For most test conditions droplets ignited within this transient region. Thus ignition delays obtained from the present study should be treated as qualitative and relative in nature, showing the global, trend-wise behavior for the range of ambient temperatures indicated. Droplets of $n$ heptane, n-hexadecane, and their mixtures, generated independently or through collision, were tested. The pure fuels were all of technical grade with purity greater than $99 \%$.

\section{Results and discussion}

Previous studies of droplet collisions [10,11,1416] used a single-component liquid for both droplets.

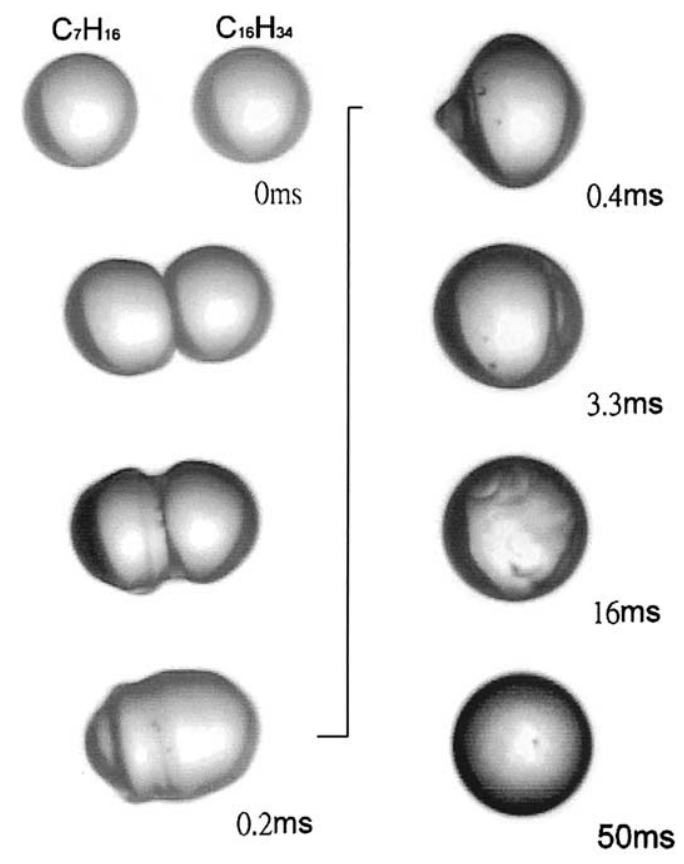

Fig. 4. Representative images showing air bubbles entrained in the coalesced droplet.

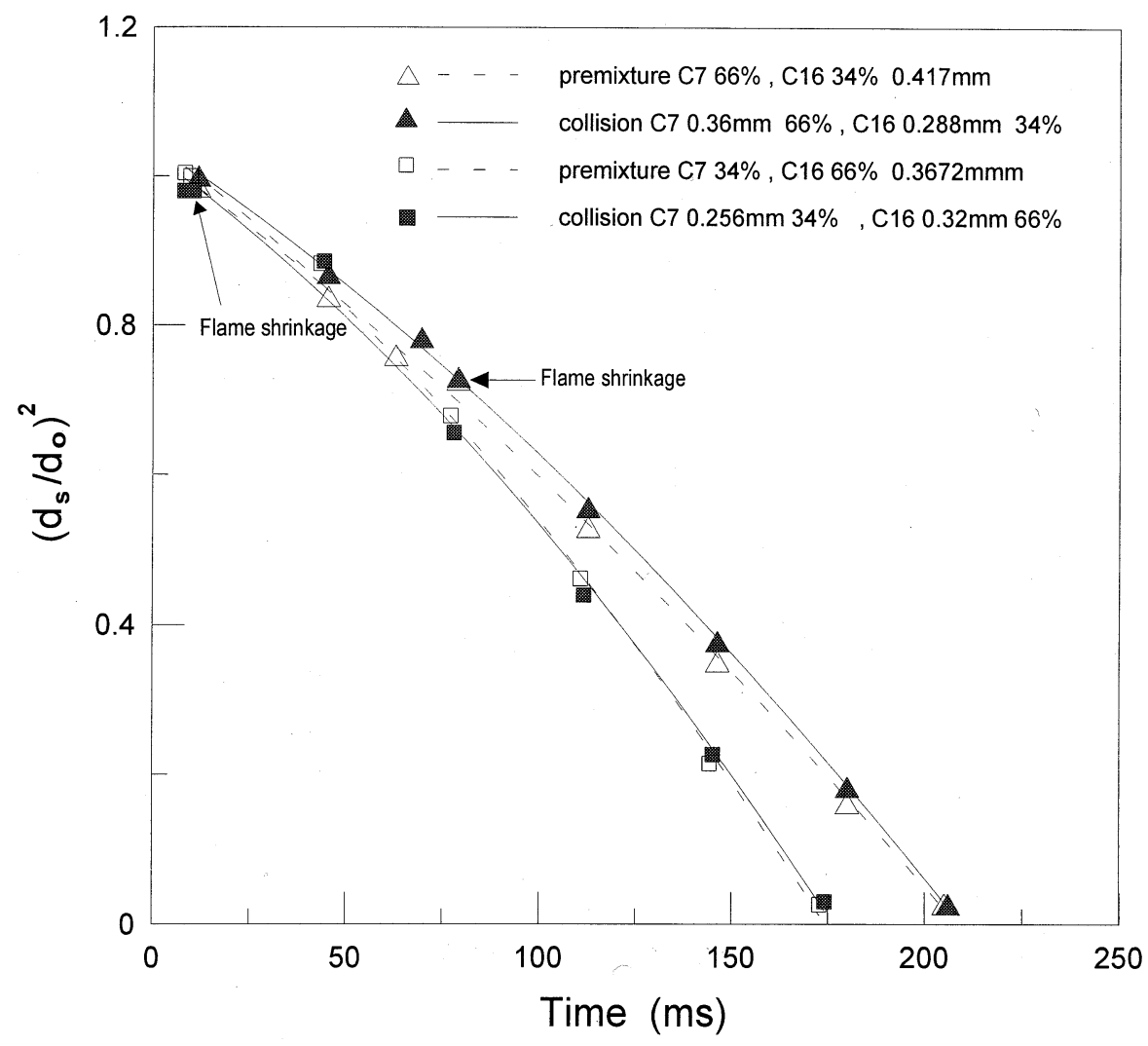

Fig. 5. Temporal variation of the square of normalized droplet diameter, $\left(\mathrm{d}_{\mathrm{s}} / \mathrm{d}_{0}\right)^{2}$. 


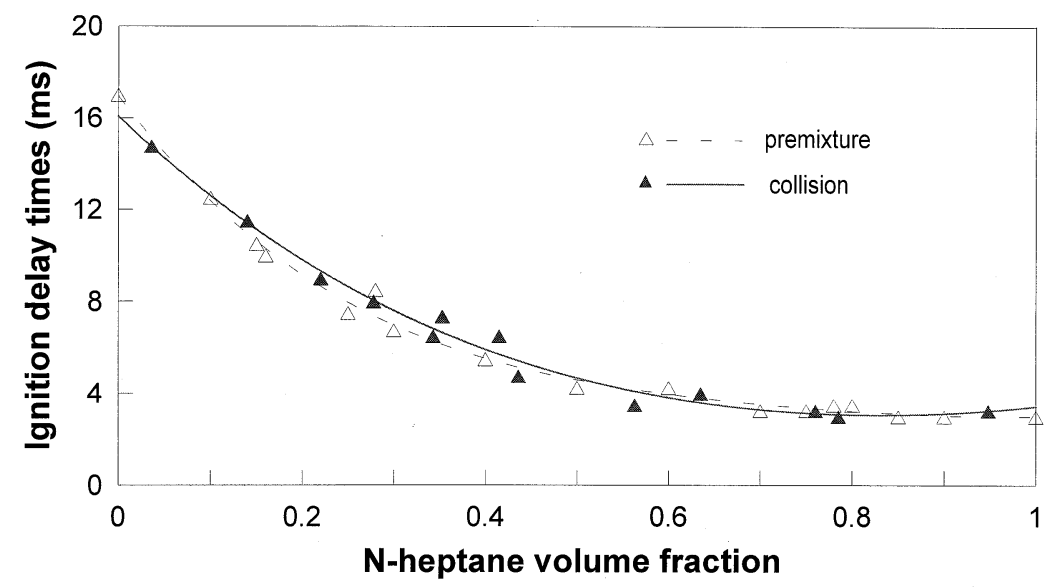

Fig. 6. Ignition delays of premixed and collision-generated heptane-hexadecane droplets.

For n-alkane droplets colliding in atmospheric air $[10,11]$, it was found that with increasing collision inertia the droplets would first merge permanently, and then bounce away, and merge permanently again, and finally merge temporarily before splitting apart with the simultaneous production of one or more satellite droplets. The present investigation of droplets of dissimilar n-alkanes showed that the collision outcomes are qualitatively the same. Because the goal of the present study was to assess the extent to which the burning of a merged droplet is affected by the uniformity of its concentration, only the collisionoutcomes characterized by permanent coalescence were examined. Figure 3 shows the photographic images of some typical collision sequences.

In general, the recorded images of the independently generated two-component droplets indicate internal uniformity. However, for collision-generated droplets, spatial non-uniformity in the transmitted light was observed during the initial period when merging just occurred, with the extent of non-uniformity being stronger with decreasing impact inertia and/or increasing off-centeredness. These observations are physically reasonable.

Of particular interest regarding the spatial nonuniformity is the presence of one or more dark spots within the merged mass (Fig. 4), indicating the possible presence of entrained bubbles of air. They were observed for collisions involving equal and unequal droplets, head-on and off-center orientations, and droplets of the same or different materials. Furthermore, some of the spots would disappear in about a few to tens of ms, possibly because of coalescence and merging, as well as through expulsion to the external environment, while they are convected and consequently exposed at the liquid surface. The presence of these dark spots has been observed before

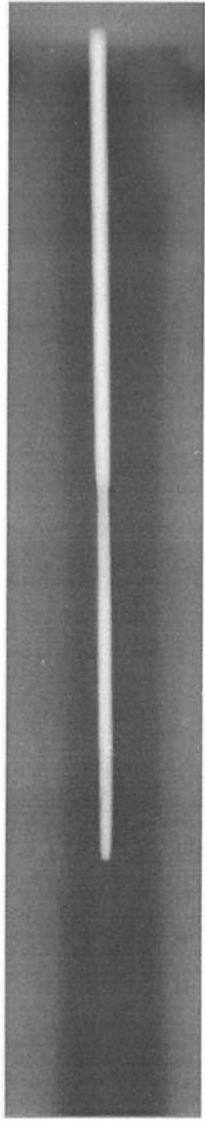

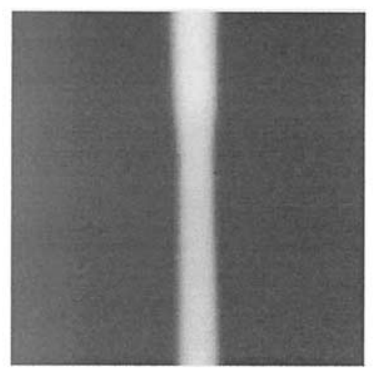

(b) (a)

Fig. 7. (a) Overall and (b) zoomed flame streaks showing the phenomenon of flame shrinkage for droplets of 80 vol.\%-heptane and 20 vol.\%-hexadecane. 


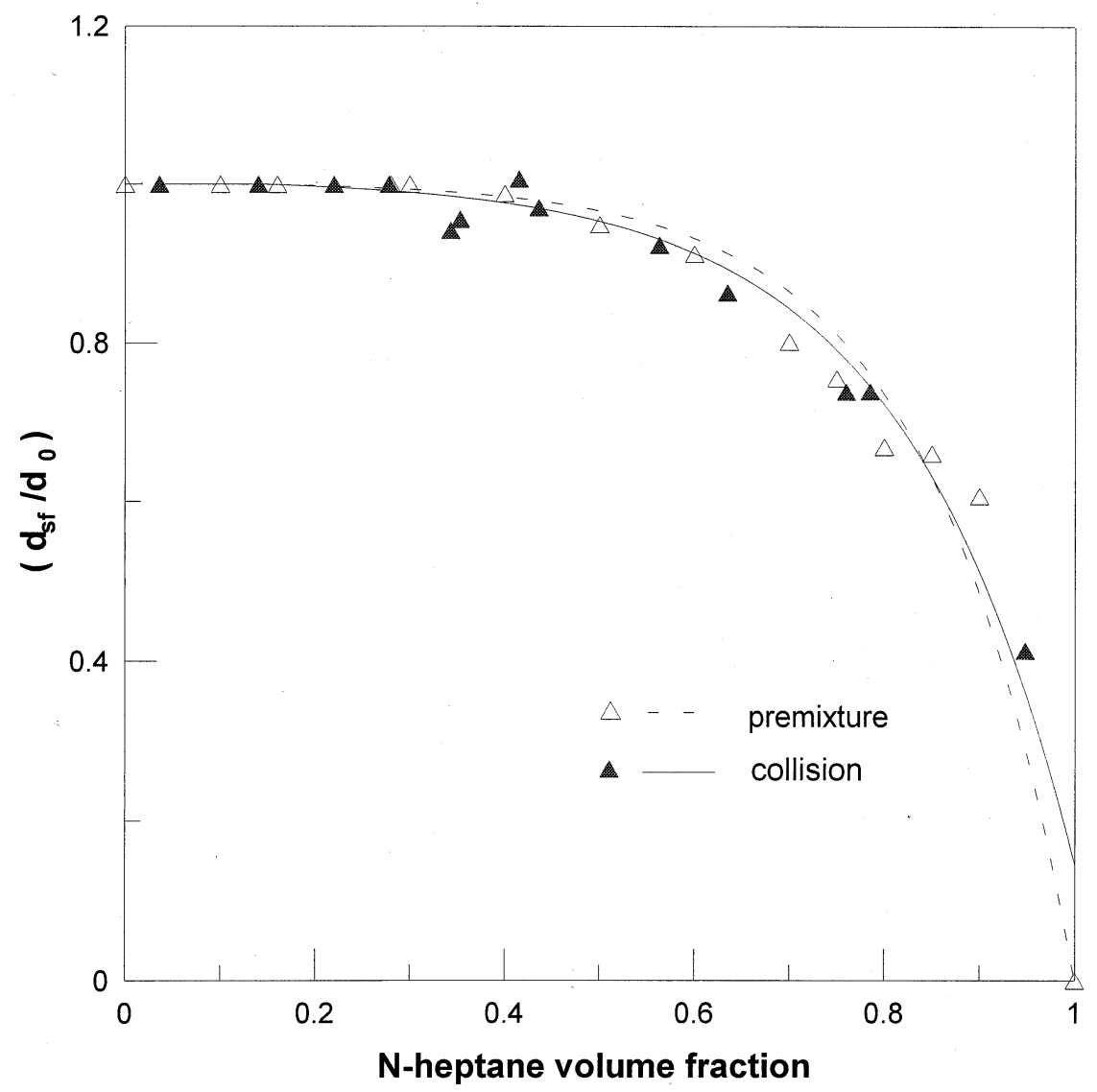

Fig. 8. Normalized droplet diameter at the state of flame shrinkage for premixed and collision-generated droplets.

$[10,15]$, with the latter [15] suggesting that they represent air bubbles trapped in the merged mass. It will be shown shortly that the occurrence of microexplosion is directly related to their presence.

It is to be emphasized that because of the repeatability and stability of the droplet stream in the experiment, the collision images as well as the data to be presented represents thousands of the coalesced droplets under basically the same collision conditions. Figure 5 plots the temporal variation of the square of the normalized droplet diameter $\left(\mathrm{d}_{\mathrm{s}} /\right.$ $\left.\mathrm{d}_{\mathrm{o}}\right)^{2}$, for both premixed and collision-generated droplets, for 34-66 and 66-34 vol.\% heptanehexadecane compositions. The figure shows that variations of the droplet size generated with the two different modes follow each other fairly closely, indicating minimal differences in their non-explosive burning characteristics in terms of, say, the ignition delay, the burning rate, and the state of flame shrinkage. These results are now discussed in more detail.

\subsection{Ignition delay}

Figure 6 shows the ignition delays for droplets of two initial compositions under fixed environmental conditions and almost the same initial droplet size of $320 \mu \mathrm{m}$. The results show that mixing within the interior of the coalesced droplet does not appear to have any significant effect on the ignition delay, and that the ignition delay decreases with the concentration of heptane. The first result indicates that, as far as ignition is concerned, the interior of the collisiongenerated droplet can be considered to be mixed during the period within which oscillation of the merged mass is damped. Because the effectiveness of damping depends on the liquid's viscosity, while the extent of molecular mixing depends on the liquid's diffusivity, and because the former is at least ten times larger than the latter, the uniformity of mixing as inferred by this ignition result should be based on the scale of the internal fluid motion. This in turn implies that the internal liquid motion generated by 
droplets merging is quite extensive and intensive, leading to large-scale uniformity within the droplet.

The second result corroborates the previous observation $[7,8]$ that the ignition delays for heptane droplets are shorter than those of hexadecane droplets. This trend is opposite to those from shock tube and flow reactor studies, which showed that, for these homogeneous systems, the ignition delays of $n$-alkanes decrease with increasing molecular size. Recognizing that the ignition delay of a droplet depends on the physical delay because of droplet heating and the chemical delay involving reaction runaway in the gas phase [17], the observations of the present study, as well as others $[7,8]$, are however consistent with the following interpretation. First, the physical delay increases with the size of the molecules, because of the increased boiling point and hence the time needed for droplet heating. For the gas-phase chemical delay, recent studies [18] on the diffusive, non-premixed counterflow ignition of a gaseous jet of an n-alkane by a heated air jet showed that the system's ignitability decreases with increasing molecular size, for molecules larger than propane. It was further demonstrated that this behavior, which is opposite to that from the homogeneous system, is a consequence of the decreased diffusivity of the larger molecules. Because droplet heating and gas-phase diffusion impose the same trend on droplet ignition, therefore, the explanation for the observed trend is unambiguous.

The final point to note is that the relative volatility of heptane and hexadecane should also play a role in the ignition delay of a two-component droplet. Specifically, because heptane is preferentially vaporized initially [3], it will facilitate ignition to a greater extent. This could explain the relatively stronger influence of heptane in reducing the ignition delay for low levels of heptane concentration.

\subsection{Flame shrinkage}

Figure 7 shows the integrated and zoomed images of the flame streaks of 80 vol.\%-heptane and 20 vol.\%-hexadecane droplet streams, demonstrating the phenomenon of flame shrinkage $[1,2,6]$ in which the flame's luminosity and size both become smaller at a certain instant of the droplet's lifetime, as mentioned earlier. Figure 8 plots the normalized flame shrinkage size, $\mathrm{d}_{\mathrm{sf}} / \mathrm{d}_{\mathrm{o}}$ versus the amount of heptane in the mixture. The result shows that there is minimal difference between the premixed and collision-generated droplets, and therefore again demonstrates the efficiency of large-scale mixing upon droplet merging. It should be noted that collision-generated droplets will either micro-explode shortly from the state of flame shrinkage or burn steadily until the very end.

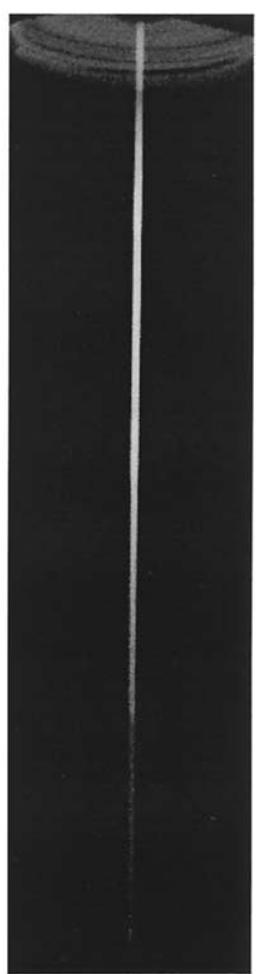

(a)

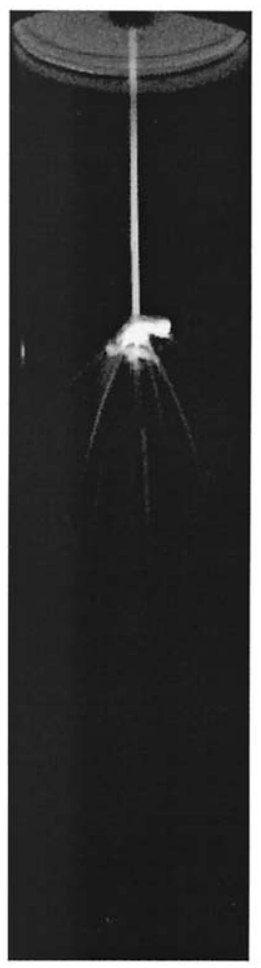

(b)
Fig. 9. Images of flame streaks for droplet burning (a) without and (b) with microexplosion.

\subsection{Microexplosion}

Figure 9 shows two flame streak images exhibiting combustion without and with microexplosion for the same initial droplet composition and size. In the present investigation microexplosion was not observed for the independently generated droplets of premixed composition. It was, however, observed for the collision-generated droplets, with the tendency increasing with impact inertia and off-centeredness, in the same manner as the presence of entrained air bubbles. For the collision-generated droplets, Fig. 10 plots the normalized droplet size at explosion, $\mathrm{d}_{\mathrm{ex}} / \mathrm{d}_{\mathrm{o}}$, against the initial concentration of heptane. Because the state of microexplosion now depends weakly on the orientation of impact, these measurements represent the maximum possible explosion size for the given composition. The results of Fig. 10 are similar to those of Lasheras et al. [4] and Wang et al. [6], showing a parabola-like dependence, with the maximum occurring around an equi-volumetric composition of 0.5 .

The present results clearly show that microexplo- 


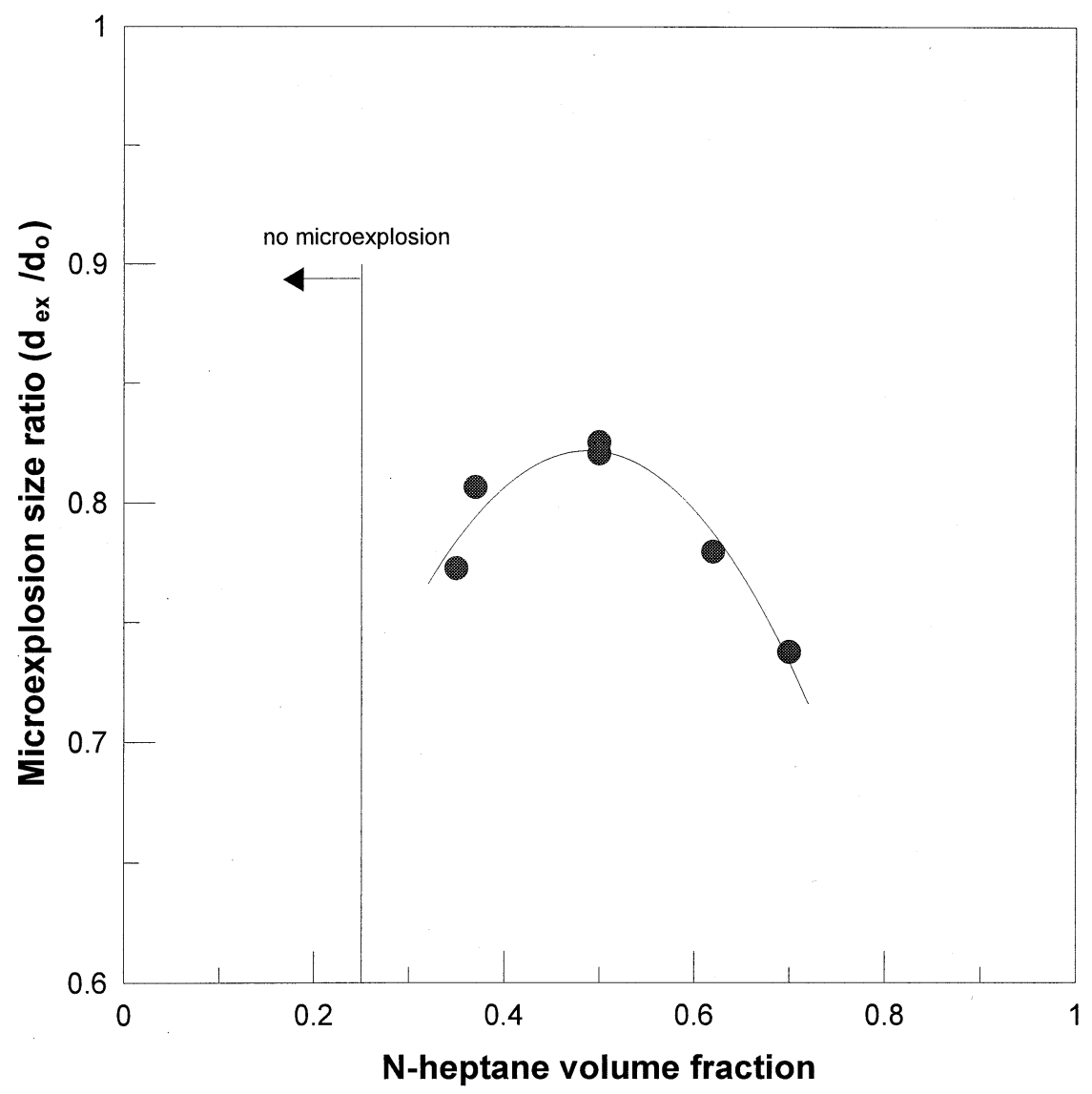

Fig. 10. Normalized droplet diameter at the state of microexplosion.

sion is not possible for burning droplets of heptane and hexadecane mixtures without internal air bubbles to facilitate nucleation. This suggestion is in accord with the following considerations. First, Wang et al. [6] reported that the occurrence of microexplosion for droplets with a volatility differential as large as that between heptane and octadecane depended on the stability of the droplet generation mode. Specifically, microexplosion was not observed if the generation mode was stable in that spherical, mono-sized droplets were readily formed at a short distance away from the nozzle. However, if the generation mode was not optimal in that the applied voltage needed to drive the piezoelectric crystal was increased from the optimal value, then a small ligament was attached to the main droplet. This ligament might or might not be absorbed by the primary droplet. If it was not absorbed, then a satellite droplet would be formed and the subsequent burning was smooth, without microexplosion. However, if it were absorbed, then microexplosion would eventually occur. It is then reasonable to suggest that air bubbles were entrained during absorption of the ligament, hence providing the needed interface for nucleation. Indeed, photographic images for the last situation did show the presence of dark spots upon droplet formation. The above interpretation is further substantiated by the results of Sangiovanni and Labowsky [13], who did not observe microexplosion for their stably generated droplets. On the other hand, the droplets of Lasheras et al. [4] were generated using a chopper to shear a liquid stream. While photographic images were not available for this process, it may be conjectured that the shearing action could produce ligaments which would entrain air bubbles upon absorption and hence induce the observed microexplosion.

\subsection{Burning rates}

Because the temporal variation of the square of the droplet diameter is not quite linear, it is practically useful to define some global burning rates to account for ignition, steady burning, and microexplosion. Based on the measured initial diameter $d_{o}$ of a 


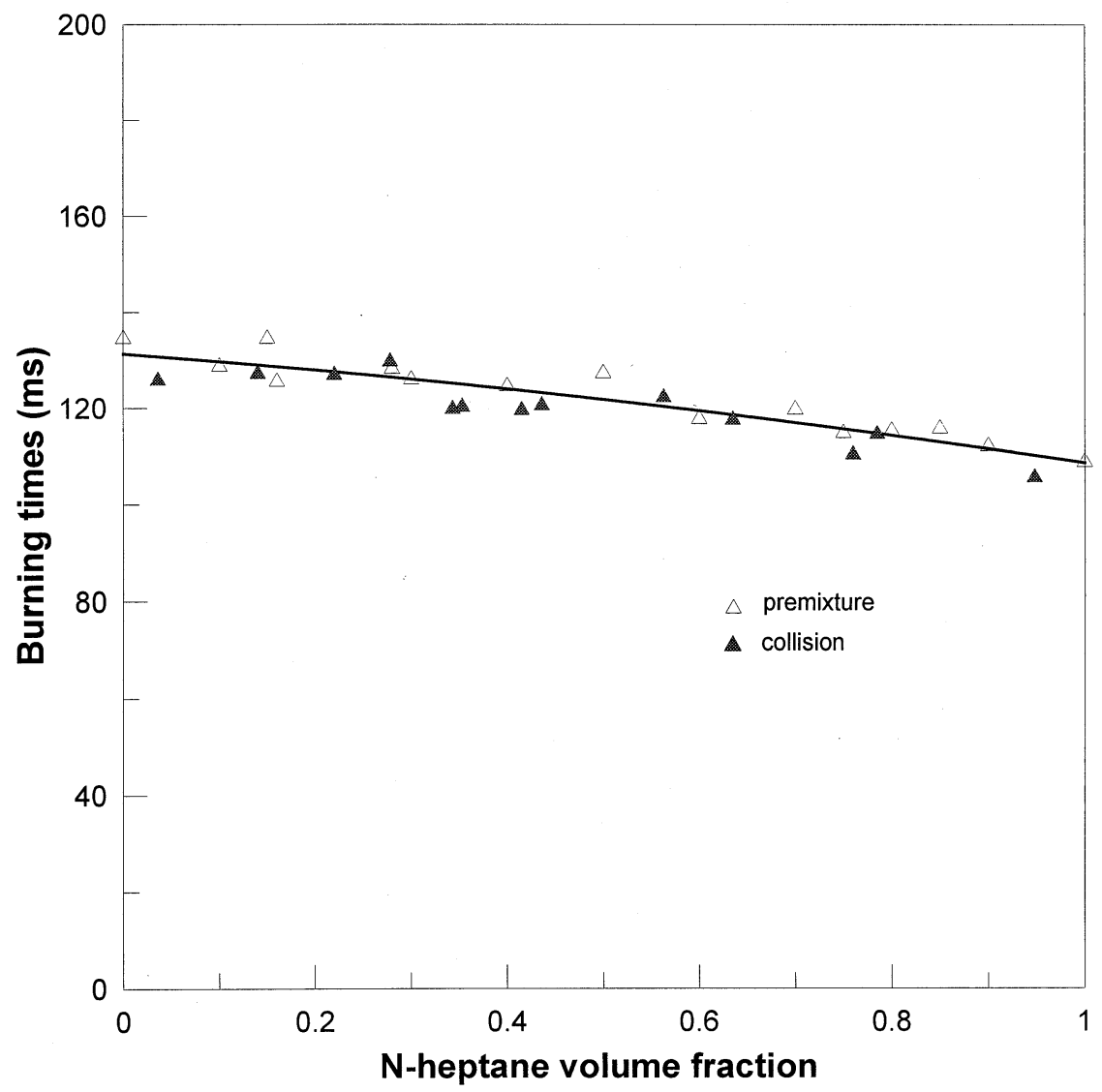

Fig. 11. Burning times of premixed and collsion-generated droplets without microexplosion.

droplet, its burning time $t_{b}$ for the period between ignition and burnout, and the overall burning time $t_{o b}$ for the period between exposure to the hot environment and burnout, an average burning rate $\mathrm{K}_{\mathrm{ab}}$ $=\mathrm{d}_{\mathrm{o}}^{2} / \mathrm{t}_{b}$ and a consumption rate $\mathrm{K}_{\mathrm{ob}}=\mathrm{d}_{\mathrm{o}}^{2} / \mathrm{t}_{\mathrm{ob}}$ can be defined. Figures 11 and 12 plot the burning times and the burning rates of non-exploding droplets generated by the two different modes, for an initial droplet diameter of $\sim 320 \mu \mathrm{m}$. It is seen that there is very little difference between results obtained with the two different generation modes, thereby further supporting the earlier observation on the ignition delay that the droplet interior can be considered to be well mixed for the collision-generated droplets at the state of ignition. Furthermore, the average burning rate increases with the concentration of heptane, while the dependence is more pronounced for the consumption rate for which the effect of the ignition delay is included.

Figure 13 shows the corresponding plots of Figs. 11 and 12, but for burning exhibiting microexplosion, with the assumption that complete liquid gasification is accomplished at microexplosion. It is seen that the occurrence of microexplosion reduces the burning time and hence increases the burning rate by approximately a factor of two, with the effect being strongest at about equi-volumetric concentrations.

\section{Conclusions}

1. The burning characteristics including the ignition delay, the burning rate, and the flame shrinkage are quite similar for two-component droplets generated either independently or through collision. This indicates that internal mixing resulting from droplets coalescencing is sufficiently intense and extensive to achieve large-scale uniformity.

2. For n-alkanes, the droplet's ignition delay decreases with increasing molecular size, in contrast to results from homogeneous ignition in shock tubes and flow reactors. For mixtures of heptane and hexadecane, the reduction is most 


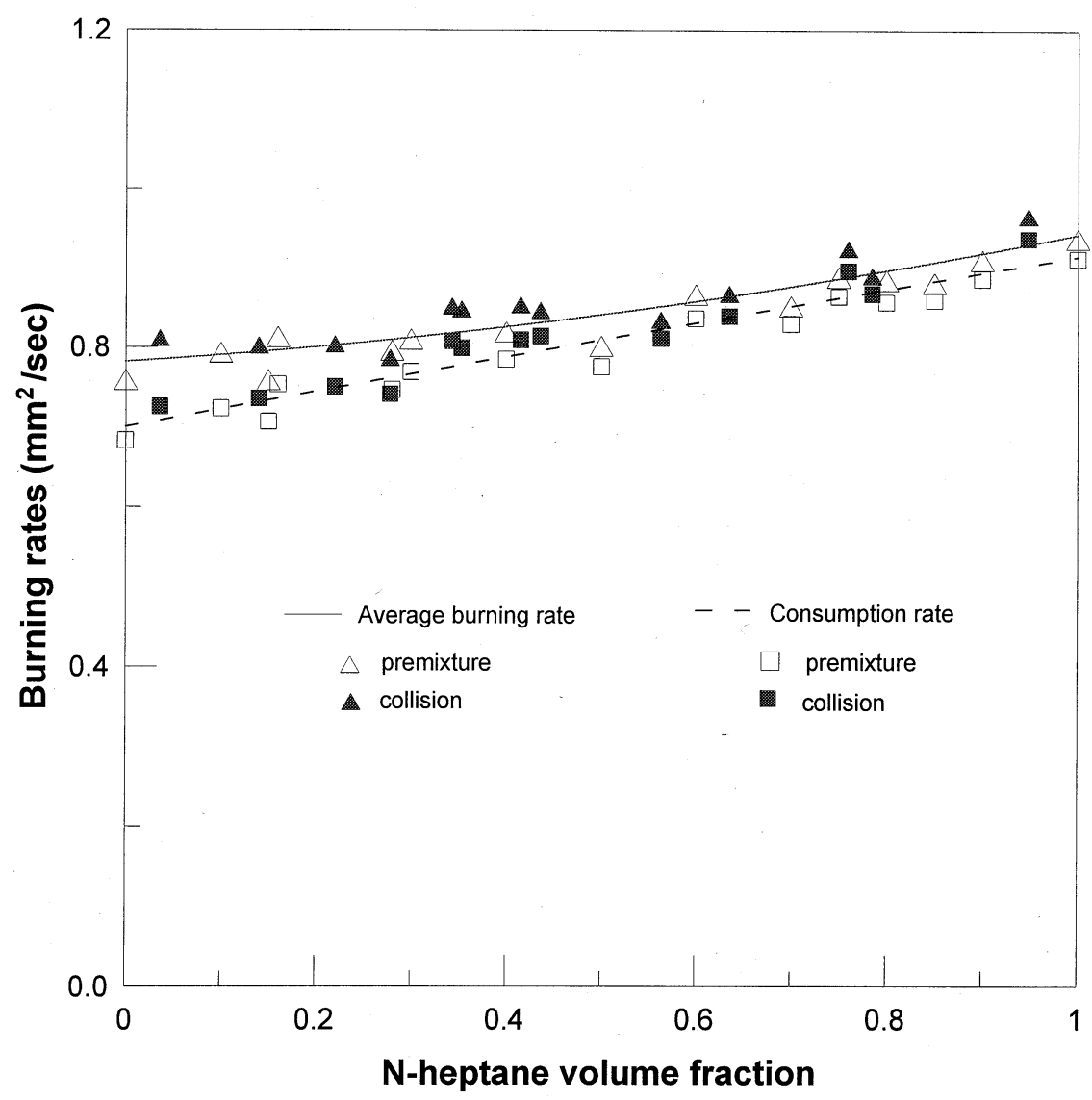

Fig. 12. Burning rates of premixed and collision-generated droplets without microexplosion.

prominent for small amounts of heptane added. Therefore, it can be suggested that the addition of a suitable amount of a volatile constituent should improve the ignitability of a mixture.

3. Because microexplosion was not observed for stably generated droplets of heptane and hexadecane, and because these two components span almost the largest volatility differential for the major components of miscible fuel blends for automotive and aero-engine applications, robust microexplosion events for droplets of these fuels are not expected without the presence of facilitating nucleation agents such as entrained bubbles.

4. The likelihood of entraining a bubble within a collision-generated droplet and hence the occurrence of droplet microexplosion increase with increasing collision velocity and off-centeredness.
5. For steadily burning droplets, the average burning rates increase with the concentration of heptane. With the aid of microexplosion, the burning rate increases sharply.

6. Because entrainment of an air bubble depends sensitively on the mode and stability of the droplet generation process, including ligament re-absorption, there exists the potential for enhanced microexplosion through manipulation of the atomization process.

\section{Acknowledgments}

The work at National Taiwan University was supported by the National Science Foundation of Taiwan, Republic of China, and at Princeton University was supported by the Air Force Office of Scientific Research under the technical monitoring of Dr. M. Birkan. 


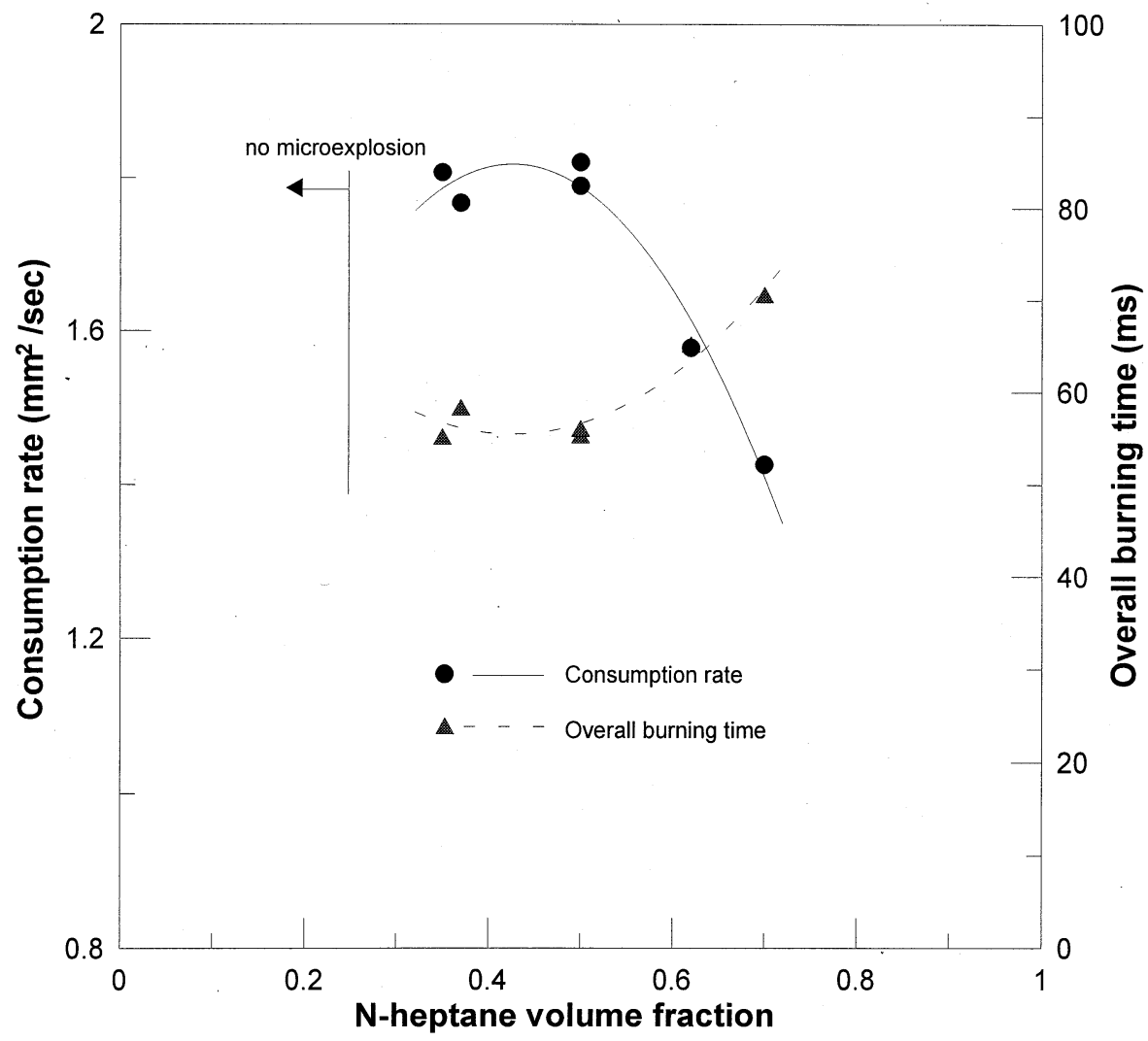

Fig. 13. Burning time and rate of collision-generated droplets with microexplosion.

\section{References}

[1] C.K. Law, Prog. Energy Combust. Sci. 8 (1982) 169.

[2] C.K. Law, H.K. Law, Modern Developments in Energy, Combustion, and Spectroscopy (F.A. Williams, A.K. Oppenheim, D.B. Olfe, M. Lapp, Eds.), Pergamon Press, Oxford, 1993, p. 29.

[3] C.K. Law, AICHE J. 24 (1978) 626.

[4] J.C. Lasheras, A.C. Fernandez-Pello, F.L. Dryer, Combust. Sci. Tech. 22 (1980) 195.

[5] J.J. Sangiovanni, A.S. Kesten, Combust. Sci. Tech. 16 (1977) 59.

[6] C.H. Wang, X.Q. Liu, C.K. Law, Combust. Flame 56 (1984) 175.

[7] C.H. Wang, K.H. Shy, L.C. Lieu, Combust. Sci. Tech. 118 (1996) 63.

[8] C.H. Wang, D.S. Liou, J. Chinese Soc. M. E. 17 (4) (1996) 387
[9] P.J. O'Rourke, F.V. Bracco, Proc. of Stratefied Charge Auto. Engineering Conf., Inst. Mech. Eng. ISBN 082984693, 1980.

[10] Y.J. Jiang, A. Umemura, C.K. Law, J. Fluid Mech. 234 (1992) 171.

[11] J. Qian, C.K. Law, J. Fluid Mech. 331 (1997) 59.

[12] J. Qian, Droplet and Flame Dynamics in Combustion Phenomena, Ph.D. Thesis, Princeton University (1997).

[13] J.J. Sangiovanni, M. Labowsky, Combust. Flame 47 (1982) 15.

[14] N. Ashgriz, P. Givi, Int. J. Heat Fluid Flow 8 (-3) (1987) 205.

[15] N. Ashgriz, J.Y. Poo, J. Fluid Mech. 221 (1990) 183.

[16] J.P. Estrade, H. Carentz, G. Lavergne, Y. Biscos, Int. J. Heat Fluid Flow 20 (1999) 486.

[17] C.K. Law, Combust. Flame 26 (1976) 219.

[18] J.D. Blouch, C.K. Law, Proceedings of the Combustion Institute, Vol. 28, The combustion Institute, Pittsburgh, (2000) p. 1679. 